\title{
"Kostenerstattung" auch durch GKV-Patienten erlaubt § 13 SGB V
}

\author{
"Kostenerstattung" bedeutet, dass der behandelnde Arzt dem Patienten die erbrachten Leistungen direkt \\ in Rechnung stellt. So ist das in der privaten Krankenversicherung (PKV) bei der Abrechnung gemäß GOÄ. \\ Im Gegensatz dazu herrscht in der gesetzlichen Krankenversicherung (GKV) das „Sachleistungsprinzip“ vor. \\ Der Patient überreicht seine Krankenversicherungskarte und erhält die medizinisch notwendigen \\ Leistungen, ohne über den monatlichen Krankenversicherungsbeitrag hinaus etwas bezahlen zu müssen. \\ Die Honorierung des Arztes dafür erfolgt über die Kassenärztliche Vereinigung mit der gesetzlichen \\ Krankenkasse. Der Patient wird üblicherweise nicht über die Höhe informiert, was im Laufe der Zeit zu \\ einer Vollkasko- oder all-inclusive-Mentalität geführt hat, die die Kosten in der GKV unnötig hochtreibt.
}

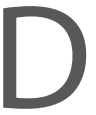
ie Möglichkeit, das Kostenerstattungssystem zu wählen, ist im SGB V im $₫ 13$ auch für Kassenpatienten festgeschrieben: Versicherte können anstelle der Sach-oder Dienstleistungen Kostenerstattung wählen ( $\$ 13, A b s$. 2). Im selben Paragrafen steht aber auch, und zwar im Absatz 1: Die Krankenkasse darf anstelle der Sach-oder Dienstleistung (\$2 Abs. 1) Kosten nur erstatten, soweit es dieses oder das Neunte Buch vorsieht. Und weiter im Absatz 2 Satz 3: Der Leistungserbringer hat die Versicherten vor Inanspruchnahme der Leistung darüber zu informieren, dass Kosten, die nicht von der Krankenkasse übernommen werden, von dem Versicherten zu tragen sind.

Die Entscheidung zur Kostenerstattung kann für die ärztliche, zahnärztliche und stationäre Behandlung und/oder veranlasste Leistungen (Arzneimittel, Heilmittel etc.) oder für eine Kombination daraus getroffen werden. Die Entscheidung gilt

Kennen Sie auch diese nützlichen DNP-Abrechnungstipps?

DNP 1/2013, Seite 27:

Neuere GOÄ-Leistungen „analog“ abrechnen

DNP 12/2012, Seite 32:

Demenzdiagnostik als IGeL - das geht

DNP 9/2012, Seite 34:

Neurologische Zusatzpauschalen -

Wann ist was möglich? jeweils für einen kompletten Bereich, das heißt, dass bei hausärztlicher Behandlung auch eine daraus resultierende notwendige fachärztliche Behandlung per Kostenerstattung abgerechnet werden muss. Der Patient ist für mindestens ein Vierteljahr an seine Entscheidung gebunden ( $\$ 13$ Abs. 2).

\section{Kostenerstattung durch GKV- Patienten darf jeder Vertragsarzt}

Im Rahmen der Kostenerstattung durch GKV-Patienten können die notwendigen Leistungen bei allen Vertragspartnern der GKV (Ärzte, Zahnärzte, Psychotherapeuten etc.) in Anspruch genommen werden. Privatärzte oder auch Physiotherapeuten ohne GKV-Vertrag können nach vorherigem Antrag mit Genehmigung durch die Krankenkasse die Behandlung beginnen. Sonstige Rechnungen (z. B. von Heilpraktikern) werden gewöhnlich nicht erstattet.

\section{Was bekommt der Patient erstattet?}

Die korrekt ausgestellte GOÄ-Rechnung, die sich nicht auf den 1-fachen Faktor beschränken muss, reicht der GKV-Patient zunächst bei seiner Krankenkasse ein. Die ist verpflichtet und berechtigt, die Kosten zu erstatten, die bei gleicher Leistung - soweit im GKV-Leistungskatalog enthalten - bei Inanspruchnahme des Sachleistungsprinzips entstanden wären. Dazu muss die Rechnung in entsprechende Leistungen des EBM transcodiert werden, was bei der derzeitigen Pauschalierung im EBM nur sehr schwer exakt möglich ist. Von dem daraus resultie- renden Betrag ist die Krankenkasse berechtigt, einen Teil als Vergütung für die anfallenden Verwaltungskosten einzubehalten. Dieser Betrag liegt je nach Krankenkasse zwischen 7,5\% und $10 \%$.

Um den Verlust für die Patienten einzugrenzen, gibt es derzeit diverse Zusatzversicherungen, die den entstehenden Differenzbetrag übernehmen. Deshalb und auch wegen des häufig vorhandenen Anspruchdenkens der Patienten ist eine Beschränkung auf den 1-fach-Satz nicht sinnvoll und oft nicht kostendeckend. Man sollte sich durchaus an seine für Privatpatienten üblichen Sätze halten. Sinnvoll ist es aber, vor der Behandlung den Patienten darauf hinzuweisen, dass seine gesetzliche Krankenkasse die Rechnung nicht komplett übernehmen wird.

\section{Fazit:}

_ Kostenerstattung ist eine legitime Art der Abrechnung von GKV-Patienten. Der Vorteil für die Praxis ist der schnellere Erhalt des Honorars, das nach den aktuell geltenden Gesetzen auch höher ist, als bei normaler GKV-Abrechnung nach dem Sachleistungsprinzip.

- Kostenerstattungspatienten sind abrechnungstechnisch keine „Schmalspur-Privatpatienten“.

- Kostenerstattungspatienten sollten vor der Behandlung darauf hingewiesen werden, dass ihre gesetzliche Krankenkasse nicht den vollen Rechnungsbetrag übernehmen kann und darf.

Dr. med. Heiner Pasch 